\title{
Autoparametric resonance of relaxation oscillations
}

\author{
F. Verhulst* and Abadi** \\ Mathematisch Instituut, University of Utrecht, POBox 80.010, 3508 TA Utrecht, The Netherlands
}

Received 26 September 2003, revised and accepted 22 June 2004

Published online 4 February

Key words relaxation oscillations, autoparametric, quenching, slow manifold.

MSC (2000) 34C26, 34D15, 37N05, 70K70

Stable normal mode vibrations in engineering can be undesirable and one of the possibilities for quenching these vibrations is by embedding the oscillator in an autoparametric system by coupling to a damped oscillator. We have the possibility of destabilising the undesirable vibrations by a suitable tuning and choice of coupling parameters. In the case of normal mode vibration derived from a relaxation oscillations we need low-frequency tuning of the attached oscillator. An additional feature is that to make the quenching effective we also have to deform the slow manifold by choosing appropriate coupling; this is illustrated for van der Pol relaxation. A number of numerical experiments have been done and show some interesting phenomena, such as a chaotic attractor and effective quenching.

(c) 2005 WILEY-VCH Verlag GmbH \& Co. KGaA, Weinheim

\section{Introduction}

Autoparametric resonance plays an important part in nonlinear engineering while posing interesting mathematical challenges. The linear dynamics is already nontrivial whereas the nonlinear dynamics of such systems is extremely rich and largely unexplored. A general characterisation of autoparametric systems is given in Tondl, Ruijgrok, Verhulst, and Nabergoj [10]. In studying autoparametric systems, the determination of stability and instability conditions of the semi-trivial solution or normal mode is always the first step. After this it is of interest to look for other periodic solutions, bifurcations and classical or chaotic limit sets.

In actual engineering problems, the loss of stability of the normal mode response depends on frequency tuning of the various components of the system, and on the interaction (the coupling) between the components. Autoparametric vibrations occur only in a limited region of the tuning parameters.

In a self-excited autoparametric system with a relaxation oscillator, to have autoparametric resonance, destabilisation of the relaxation oscillation of the system is needed. It turns out that to destabilize relaxation oscillations one needs in addition rather strong interactions of a special form. This is tied in with the necessity to perturb the slow manifold which characterizes to a large extent the relaxation oscillation. The results in this paper are an extension of Verhulst [12] but are, regarding the numerical experiments of the last sections, still mainly exploratory.

The monograph by Tondl et al. [10] contains a survey of the literature on self-excited autoparametric systems, in particular for weak self-excitation and weak interactions; see also Schmidt and Tondl [9] and Cartmell [2].

\section{Formulation of the problem}

A typical formulation for autonomous systems runs as follows. Consider the one-degree-of-freedom, i.e., two-dimensional system

$$
\dot{x}=f(x)
$$

where $f(x)$ is a smooth 2-dimensional vector field and assume that the equation has a stable periodic solution. Suppose that this corresponds with undesirable behaviour, as is for instance the case of flow-induced vibrations. Can we introduce a kind of energy absorber, mathematically speaking can we couple the equation to another system such that this periodic solution

\footnotetext{
* Corresponding author, e-mail: verhulst@math.uu.nl

** On leave from the Department of Mathematics, Surabaya State University (UNESA), Indonesia.
} 
arises as an unstable normal mode in the full system? This entails the introduction of the system

$$
\begin{aligned}
\dot{x} & =f(x)+g(x, y), \\
\dot{y} & =h(x, y),
\end{aligned}
$$

in which $y$ is $n$-dimensional, $g$ and $h$ are smooth vector fields, with $h(x, 0)=0$. In most cases we assume $g(x, 0)=0$, so that the original periodic solution corresponds with a normal mode of the coupled system. Sometimes $g(x, y)$ includes a perturbation resulting in a normal mode close to the unperturbed one. The important questions are 'what are the requirements for the coupling terms $g$ and $h$ to achieve effective destabilisation of the normal mode' and 'how do we choose the system parameters'.

Suppose $\phi(t): \mathbb{R} \rightarrow \mathbb{R}^{2}$ is a stable $T$-periodic solution of the equation

$$
\dot{x}=f(x)+g(x, 0) .
$$

We shall study the stability of this normal mode in system (1).

\section{Linearisation and decoupling}

We put $x=\phi(t)+u, y=y$ and expand to obtain the linearised system

$$
\begin{aligned}
& \dot{u}=\frac{\partial f}{\partial x}(\phi(t)) u+\frac{\partial g}{\partial x}(\phi(t), 0) u+\frac{\partial g}{\partial y}(\phi(t), 0) y, \\
& \dot{y}=h(\phi(t), 0) .
\end{aligned}
$$

With a slight abuse of notation we kept $u$ and $y$ for the solutions of the linear system. It is clear that the linear system is decoupled in the following sense. The equation for $y$ can be studied independently with the requirement to produce instability. Subsequently in the equation for $u$ the behaviour of $y$ can be introduced to study the behaviour of $u$.

It follows from Poincaré-Lyapunov theory (see [11]) that if $y=0$ is unstable for the second equation, the normal mode is unstable. The instability becomes however more effective for our purpose if also the solution $u$ of the first equation is unstable.

The homogeneous part of the first equation of (2) reads

$$
\dot{v}=\frac{\partial f}{\partial x}(\phi(t)) v+\frac{\partial g}{\partial x}(\phi(t), 0) v,
$$

which is a linear equation with $T$-periodic coefficients. One of the solutions is $\dot{\phi}(t)$ and we can easily construct a second independent solution by d'Alembert's method. What interests us, however, are the characteristic (or Floquet- or Lyapunov-) exponents. The exponent corresponding with $\dot{\phi}(t)$ is of course zero, as this solution is periodic. The second exponent, $\lambda$, is negative by assumption and reads

$$
\lambda=\frac{1}{T} \int_{0}^{T} \operatorname{Tr}\left(\frac{\partial f}{\partial x}(\phi(t))+\frac{\partial g}{\partial x}(\phi(t), 0)\right) d t .
$$

For a proof of these classical statements see for instance Verhulst [11]. This result can now be used to study the stability of the trivial solution of the equation for $u$ where the inhomogeneous part $u_{i}(t)$ may destabilize $u=0$.

It follows from Floquet-theory that the second independent solution is of the form $\mathrm{e}^{-\lambda t} \psi(t)$, where $\psi(t)$ is $T$-periodic. With fundamental matrix $\Theta(t)=\left(\dot{\phi}(t), \mathrm{e}^{-\lambda t} \psi(t)\right)$, the inhomogeneous part of the solution for $u$ becomes

$$
u_{i}(t)=\Theta(t) \int_{0}^{t} \Theta^{-1}(s) \frac{\partial g}{\partial y}(\phi(s), 0) y(s) d s .
$$

It is clear from this expression that the growth of $y(t)$ - the instability of $y=0$ - is a necessary condition for the instability of $u=0$. Whether this condition is sufficient depends on the actual autoparametric system as we shall see in the applications.

Often the first (vector-)equation of (2) can be written as a scalar second order equation of the form

$$
\ddot{u}+p(t) \dot{u}+q(t) u=F(t) y
$$

with scalar independent (homogeneous) solutions $\dot{\phi}(t)$ and $\mathrm{e}^{-\lambda t} \psi(t)$ and the Wronskian $\mathrm{e}^{-\lambda t} \chi(t), \chi(t)$ a $T$-periodic function. In this case a particular solution of the inhomogeneous equation (4) reads

$$
u_{i}(t)=\dot{\phi}(t) \int_{0}^{t} \frac{\psi(s)}{\chi(s)} F(s) y(s) d s-\mathrm{e}^{-\lambda t} \psi(t) \int_{0}^{t} \frac{\mathrm{e}^{\lambda s}}{\chi(s)} \dot{\phi}(s) F(s) y(s) d s .
$$

Note that $\chi(t)$ has no zeros. 


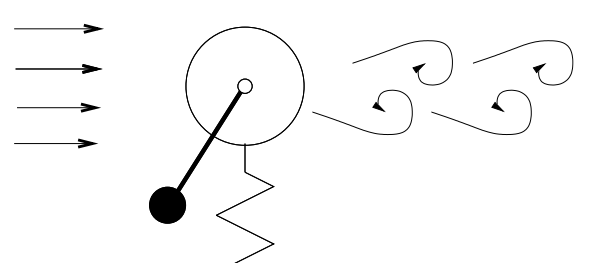

Fig. 1 Example of an autoparametric system with flow-induced vibrations. The system consists of a single mass on a spring to which a pendulum is attached as an energy absorber. The flow excites the mass and the spring but not the pendulum.

\section{Weak coupling of self-excited oscillations}

For reasons of comparison with the results in the sequel we make some observations about weak self-excitation and weak interaction. We shall express these by using the small, positive parameter $\varepsilon$.

Consider the case of flow-induced vibrations represented by the Rayleigh oscillator embedded in the autoparametric system (different from the example in the figure)

$$
\begin{aligned}
\ddot{x}+x & =\varepsilon\left(1-\dot{x}^{2}\right) \dot{x}+\varepsilon\left(c_{1} x^{2}+c_{2} x y+c_{3} y^{2}\right), \\
\ddot{y}+\varepsilon \kappa \dot{y}+q^{2} y & =\varepsilon y\left(d_{1} x+d_{2} y\right) .
\end{aligned}
$$

The damping coefficient $\kappa$ is positive, the frequency $q$ and the coefficients $c_{i}, d_{i}$ will be chosen suitably, i.e., to provide optimal instability of the normal mode $\phi(t)$ obtained by putting $y=0$. The $T$-periodic solution $\phi(t)$ corresponds with self-excited vibrations and we linearise around this normal mode, putting $x=\phi(t)+u, y=y$, to find:

$$
\begin{aligned}
\ddot{u}+u & =\varepsilon\left(1-3 \dot{\phi}(t)^{2}\right) \dot{u}+\varepsilon\left(2 c_{1} \phi(t) u+c_{2} \phi(t) y\right), \\
\ddot{y}+\varepsilon \kappa \dot{y}+q^{2} y & =\varepsilon d_{1} \phi(t) y .
\end{aligned}
$$

The equation for $y$ is Hill's equation with damping added which can be reduced to Mathieu's equation by using that $\varepsilon$ is small.

It is well known that we have for the periodic solution of the modified Rayleigh oscillator

$$
\ddot{x}+x=\varepsilon\left(1-\dot{x}^{2}\right) \dot{x}+\varepsilon c_{1} x^{2}
$$

the approximation

$$
\phi(t)=2 \cos (t)+O(\varepsilon) .
$$

The estimate for amplitude and period is valid for all time. Inserting this into the equation for $y$ yields

$$
\ddot{y}+\varepsilon \kappa \dot{y}+\left(q^{2}-2 \varepsilon d_{1} \cos (t)\right) y=0 .
$$

In parameter space a relatively large instability domain arises on choosing $q=\frac{1}{2}$. The usual analysis (Poincaré-Lindstedt, averaging or harmonic balance) leads to the (known) requirement $\left|d_{1}\right|>\frac{1}{2} \kappa$ for instability of $y=0$.

Returning to the equation for $u$ we note that we have from eq. (3) and the first equation of (6)

$$
\lambda=\varepsilon \frac{1}{2 \pi} \int_{0}^{2 \pi}\left(1-3 \dot{\phi}(s)^{2}\right) d s=-5 \varepsilon+O\left(\varepsilon^{2}\right) .
$$

Independent solutions of the homogeneous part of the first equation of (6) are according to Floquet theory $\dot{\phi}(t)$ and $\mathrm{e}^{-5 \varepsilon t} \psi(t)+O(\varepsilon)$ with $\psi(t)$ a $T$-periodic solution which can be obtained by d'Alembert's construction. The Wronskian to $\mathcal{O}(\varepsilon)$ becomes $\mathrm{e}^{-5 \varepsilon t} \chi(t)$ with again $\chi(t)$ a $T$-periodic function without zeros. The particular solution of the equation for $u$ takes the form

$$
u_{i}(t)=\varepsilon c_{2} \dot{\phi}(t) \int_{0}^{t} \frac{\psi(s) \phi(s)}{\chi(s)} y(s) d s-\varepsilon c_{2} \mathrm{e}^{-5 \varepsilon t} \psi(t) \int_{0}^{t} \mathrm{e}^{5 \varepsilon s} \frac{\dot{\phi}(s) \phi(s)}{\chi(s)} y(s) d s .
$$

We conclude that on choosing $q=\frac{1}{2}, d_{1}>\frac{1}{2} \kappa$ the solution $y=0$ becomes unstable which destabilises the normal mode in the $y$-direction. On choosing $c_{2} \neq 0$ the solution $u=0$ also becomes unstable which enforces the instability of the normal mode. The parameters $c_{1}, c_{3}, d_{2}$ play no part at this level of approximation. 
Note that Abadi [1] studied this autoparametric system in the case $c_{1}=c_{2}=d_{2}=0$ with emphasis on the bifurcation phenomena in the case of an unstable normal mode.

We mention that in the case of weak interaction the analysis does not change much when we replace the Rayleigh oscillator by van der Pol self-excitation. The Lyapunov exponents of the normal mode in this case are 0 and $\lambda=-\varepsilon+O\left(\varepsilon^{2}\right)$.

\section{Interaction with relaxation oscillations}

A different problem arises when we wish to quench a relaxation oscillation, i.e., when we wish to reduce its amplitude(s). We take as an example the van der Pol relaxation oscillator embedded in an autoparametric system of the form

$$
\begin{aligned}
\ddot{x}+x & =\mu\left(1-x^{2}\right) \dot{x}+F(x, \dot{x}, y, \dot{y}), \\
\ddot{y}+\kappa \dot{y}+q^{2} y & =y G(x, \dot{x}, y, \dot{y}),
\end{aligned}
$$

where $\kappa$ is again a positive damping coefficient. We assume that if the $(y, \dot{y})$-vibration is absent, $F$ vanishes to produce a pure vander Pol relaxation oscillation: $F(x, \dot{x}, 0,0)=0$. The functions $F$ and $G$, and the remaining parameters have to be chosen to produce instability of the (periodic relaxation) normal mode, obtained by putting $y=0$ in the case $\mu \gg 1$. We will use results on this relaxation oscillator which were summarised and extended by Grasman [7]. We will also use results on slow manifolds in geometric singular perturbations; for an introduction see Kaper [8] and the original papers by Fenichel [3-6].

Introducing $\phi(t)$ for the $T_{\mu}$-periodic relaxation normal mode, putting $x=\phi(t)+u, y=y$ produces

$$
\begin{aligned}
\ddot{u}+u & =\mu\left(1-\phi(t)^{2}\right) \dot{u}-2 \mu \phi(t) \dot{\phi}(t) u+\ldots, \\
\ddot{y}+\kappa \dot{y}+\left(q^{2}-d_{1} \phi(t)\right) y & =\ldots,
\end{aligned}
$$

where the nonlinear terms are indicated by dots. The linearised equations suffice for the stability analysis.

\section{The Lyapunov exponent of relaxation}

The normal mode is $T_{\mu}$-periodic with the estimate (see Grasman [7])

$$
T_{\mu}=(3-2 \log 2) \mu+O\left(\frac{1}{\mu^{\frac{1}{3}}}\right) \quad \text { as } \quad \mu \rightarrow \infty .
$$

To compute the rate of attraction from the integral in (3) we use the slow-fast motion in the Liénard plane by replacing the van der Pol equation by

$$
\dot{x}=\mu\left(z+x-\frac{1}{3} x^{3}\right), \quad \mu \dot{z}=-x .
$$

In the $x-z$ Liénard plane we have

$$
\left(z+x-\frac{1}{3} x^{3}\right) \frac{d z}{d x}=-\frac{x}{\mu^{2}},
$$

which illustrates that as $\mu$ is large $d z / d x$ is very small except if $z=-x+\frac{1}{3} x^{3}$. This cubic curve in the Liénard plane corresponds with the slow manifold of the system. See Fig. 2.

With this knowledge it is not difficult to obtain a first order approximation of the characteristic exponent. From eq. (3) and the first equation of (9), we have

$$
\lambda=\mu \frac{1}{T_{\mu}} \int_{0}^{T_{\mu}}\left(1-\phi(t)^{2}\right) d t .
$$

Integration of van der Pol's equation for the periodic solution yields

$$
\int_{0}^{T_{\mu}}(\ddot{\phi}(t)+\phi(t)) d t=\mu \int_{0}^{T_{\mu}}\left(1-\phi^{2}(t)\right) \dot{\phi}(t) d t
$$


or, using the periodicity,

$$
\int_{0}^{T_{\mu}} \phi(t) d t=\mu \oint\left(1-x^{2}\right) d x=0,
$$

where the contour integral is taken over the limit cycle in the phase plane. With this result, using partial integration and the equation for $z$ we have

$$
\int_{0}^{T_{\mu}} \phi(t)^{2} d t=-\int_{0}^{T_{\mu}}\left(\int \phi(t) d t\right) \dot{\phi}(t) d t=\mu \oint z(x) d x .
$$

Integration in the Liénard plane yields the approximation

$$
\lambda=\mu\left(1-\frac{9}{2} \frac{\mu}{T_{\mu}}\right)+o(1) \approx-1.79 \mu,
$$

which, as $\mu \gg 1$, corresponds with strong attraction.

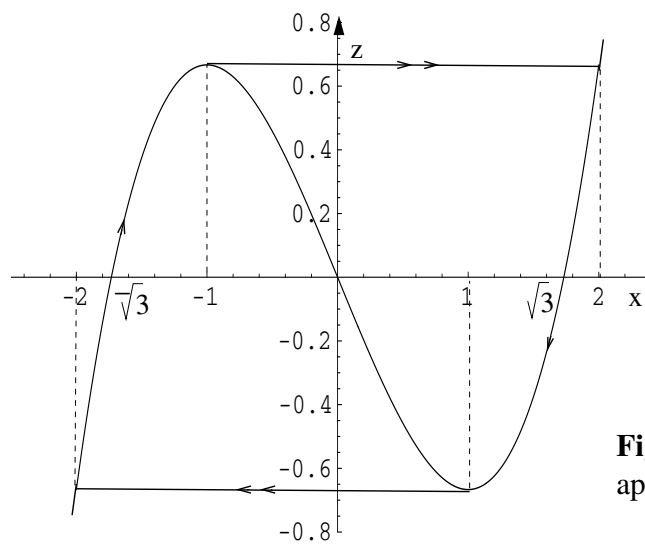

Fig. 2 The phase plane of the van der Pol relaxation oscillation. The slow manifold is approximated by the cubic curve, fast motion is indicated by double arrows.

\section{Instability of $y=\dot{y}=0$}

The periodic coefficient $\phi(t)$ in the equation for $y$ has a period proportional to $\mu \gg 1$, so it is natural to rescale $t=\frac{T_{\mu}}{2 \pi} \tau$ which, after linearisation, produces

$$
\frac{d^{2} y}{d \tau^{2}}+\frac{\kappa T_{\mu}}{2 \pi} \frac{d y}{d \tau}+\left(\frac{q^{2} T_{\mu}^{2}}{4 \pi^{2}}-\frac{d_{1} T_{\mu}^{2}}{4 \pi^{2}} \phi_{r}(\tau)\right) y=0
$$

with $\phi_{r}(\tau)=\phi\left(\frac{T_{\mu}}{2 \pi} \tau\right), 2 \pi$-periodic in $\tau$. We observe that the instability behaviour of the solutions of the Floquet equation (10) is qualitatively the same as for the damped Mathieu-equation. A consequence is that to obtain prominent instability of $y=0$ and so destabilisation of the relaxation oscillation we have to couple to a low-frequency oscillator $(y)$ with, using the first-order estimate for the period,

$$
\kappa=O\left(\frac{1}{\mu}\right), \quad q=\frac{\pi}{(3-2 \log 2) \mu}, \quad d_{1}=O\left(\frac{1}{\mu^{2}}\right) \quad \text { as } \quad \mu \rightarrow \infty .
$$

The actual choice of $\kappa$ and $d_{1}$ depends on the amount of quenching one wants to achieve. We explore the small parameter case

$$
\frac{\kappa T_{\mu}}{2 \pi}=\frac{\kappa_{0}}{\mu}, \quad \frac{q^{2} T_{\mu}^{2}}{\pi^{2}}=1, \quad \frac{d_{1} T_{\mu}^{2}}{4 \pi^{2}}=\frac{d}{\mu}
$$

with $\kappa_{0}$ and $d$ independent of $\mu$. We have from eq. (10)

$$
\frac{d^{2} y}{d \tau^{2}}+\frac{\kappa_{0}}{\mu} \frac{d y}{d \tau}+\left(\frac{1}{4}-\frac{d}{\mu} \phi_{r}(\tau)\right) y=0 .
$$


Inside the corresponding Floquet-tongue the solutions are unstable, outside they are stable. On the boundary of the Floquettongue the solutions are periodic, see for instance [11], in particular the treatment of the Mathieu-equation in appendix 2. So, to obtain the boundaries of the Floquet-tongue we impose the periodicity conditions

$$
\begin{aligned}
& \int_{0}^{2 \pi}\left(-\kappa_{0} \frac{d y(\tau)}{d \tau}+d \phi_{r}(\tau)\right) y(\tau) \sin \frac{\tau}{2}=0 \\
& \int_{0}^{2 \pi}\left(-\kappa_{0} \frac{d y(\tau)}{d \tau}+d \phi_{r}(\tau)\right) y(\tau) \cos \frac{\tau}{2}=0
\end{aligned}
$$

With the Poincaré expansion $y(\tau)=a_{0} \cos \frac{\tau}{2}+b_{0} \sin \frac{\tau}{2}+\frac{1}{\mu} \ldots$, we have

$$
\frac{1}{2} \kappa_{0} a_{0}-d I b_{0}=0, \quad d I a_{0}-\frac{1}{2} \kappa_{0} b_{0}=0
$$

where we used the symmetry of $\phi_{r}(\tau) ; I$ is a positive number given by

$$
I=\int_{0}^{2 \pi} \phi_{r}(\tau) \cos \tau d \tau
$$

Nontrivial solutions arise if the determinant vanishes or

$$
\frac{1}{2} \kappa_{0}= \pm d I
$$

We have instability of $y=0$ if $|d|>\frac{\kappa_{0}}{2 I}$. This is the analogue of the result obtained in Sect. 4 for weak interaction.

\section{Deformation of the slow manifold}

To analyse the relaxation oscillation in the 4-dimensional problem of system (8) we assume that the interaction term $F$ contains quadratic and cubic terms and is of the form

$$
F(x, \dot{x}, y, \dot{y})=\mu\left(c_{1} \dot{x} y+c_{2} x \dot{x} y+c_{3} \dot{x} y^{2}\right) .
$$

An easy way to see that these are the leading terms of $F$ runs as follows. Transform the time in the first equation of (8) $t \rightarrow \mu \tau$ and indicate differentiation with respect to $\tau$ with a prime:

$$
\frac{1}{\mu^{2}} x^{\prime \prime}+x=\left(1-x^{2}\right) x^{\prime}+F\left(x, \frac{1}{\mu} x^{\prime}, y, \frac{1}{\mu} y^{\prime}\right)
$$

or

$$
x^{\prime}=v, \quad \frac{1}{\mu^{2}} v^{\prime}=\left(1-x^{2}\right) v+F\left(x, \frac{1}{\mu} v, y, \frac{1}{\mu} y^{\prime}\right) .
$$

The slow manifold is obtained by putting the right-hand side of the equation for $v$ to zero. For $F$ to induce a significant deformation its terms have to depend on $v$ but this produces a factor $\frac{1}{\mu}$; terms containing $v^{2}$ or $v y^{\prime}$ produce terms of order $\frac{1}{\mu^{2}}$ and can be omitted.

As an illustration we choose for the attached $(y)$ oscillator $G=d x$.

For the van der Pol relaxation oscillator the Liénard transformation is useful, see [7]. We generalize the Liénard transformation $(x, \dot{x}) \rightarrow(x, z)$ for our problem to

$$
\begin{aligned}
\frac{1}{\mu} \dot{x} & =z+x-\frac{1}{3} x^{3}+c_{1} x y+\frac{1}{2} c_{2} x^{2} y+c_{3} x y^{2}, \\
\dot{z} & =-\frac{1}{\mu} x-c_{1} x \dot{y}-\frac{1}{2} c_{2} x^{2} \dot{y}-2 c_{3} x y \dot{y} .
\end{aligned}
$$

The slow manifold is given by

$$
z=-x+\frac{1}{3} x^{3}-c_{1} x y-\frac{1}{2} c_{2} x^{2} y-c_{3} x y^{2} .
$$


It is unstable if

$$
1-x^{2}+c_{1} y+c_{2} x y+c_{3} y^{2}>0 .
$$

The $c_{3}$-term is semidefinite, which is important, as far as stability is concerned. So, we choose this term for our model of destabilisation of the relaxation oscillation. Replacing $c_{3}$ by $c$ we have the system

$$
\begin{aligned}
\ddot{x}+x & =\mu\left(1-x^{2}\right) \dot{x}+\mu c \dot{x} y^{2}, \\
\ddot{y}+\kappa \dot{y}+q^{2} y & =d x y .
\end{aligned}
$$

In generalised Liénard variables this becomes

$$
\begin{aligned}
\frac{1}{\mu} \dot{x} & =z+x-\frac{1}{3} x^{3}+c x y^{2} \\
\dot{z} & =-\frac{1}{\mu} x-2 c x y \dot{y}
\end{aligned}
$$

with the equation for $y$ added. The slow manifold is given by

$$
z=-\left(1+c y^{2}\right) x+\frac{1}{3} x^{3}
$$

which is unstable if $1+c y^{2}-x^{2}>0$. The slow manifold corresponds with a 3-dimensional cubic cylinder parallel to the $\dot{y}$-axis.

We are now able to illustrate the behaviour of this autoparametric system.

\section{Numerical experiments}

At present the dynamics of system (14) in the case of an unstable normal mode is far from clear. In anticipation of a more theoretical analysis in the near future we perform a number of numerical experiments to illustrate interesting phenomena. We choose $\mu=10$ throughout.

The stability of the slow manifold is determined by the sign of $1+c y^{2}-x^{2}$. We shall take $c$ negative, $c=-2.2$, to illustrate the effect of a growing $y$-oscillation. In this case $1+c y^{2}=0$ if $y=0.67 \ldots$ Fig. 3 describes the slow manifold and the corresponding stability manifold.
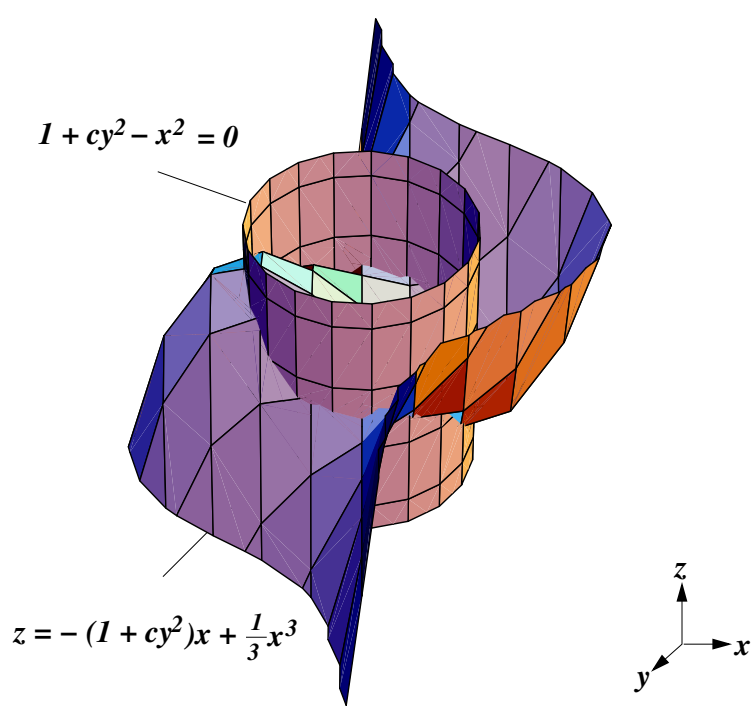

Fig. 3 (online colour at: www.zamm-journal.org) The slow manifold and the stability manifold for $c=-2.2$. The slow manifold is a 3 -dimensional cubic cylinder in the complete 4-dimensional phase space. The stability of the flow on the slow manifold is determined by its position with respect to the manifold given by $1+c y^{2}-x^{2}=0$.

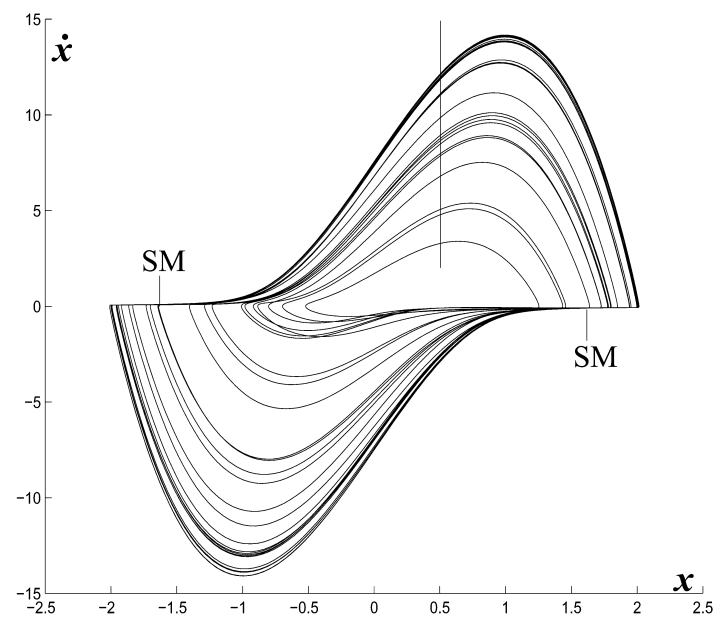

Fig. 4 A limit set of system (14) for $\mu=10, c=$ $-2.2, d=0.03, \kappa=0.075$ with small starting values of the $y$-oscillation, projected on the $x-\dot{x}$ plane. $S M$ is the stable part of the slow manifold. The vertical line corresponds with the Poincaré section of Fig. 5. 

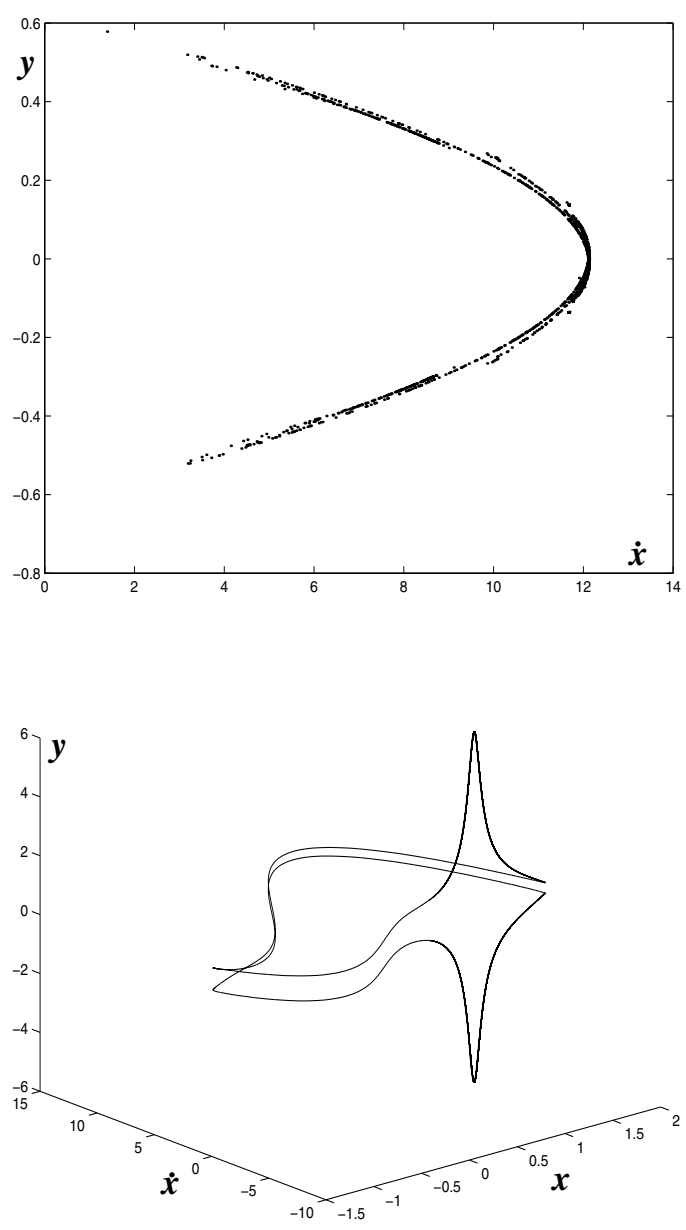

Fig. 6 A periodic limit set of system (14) for $\mu=$ $10, c=-2.2, d=0.03, \kappa=0.075$ with high starting values of the $y$-oscillation. Transient orbits are left out. The stable part of the slow manifold is present near the extreme values of $y$.
Fig. 5 The Poincaré section of the limit set of Fig. 4 projected on the $\dot{x}-y$ plane.

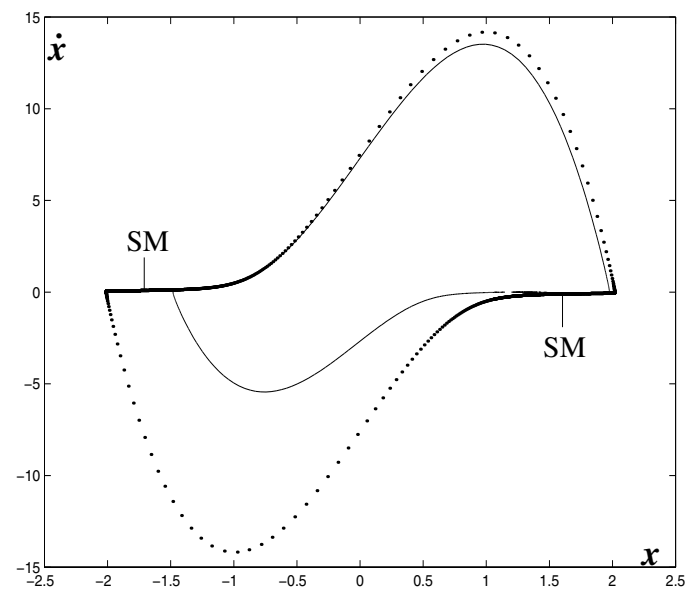

Fig. 7 A periodic limit set of system (14) for $\mu=10, c=-2.2, d=$ $0.03, \kappa=0.075$ with high starting values of the $y$-oscillation, projected on the $x-\dot{x}$ plane. The dotted orbit corresponds with the unperturbed relaxation oscillation. In the perturbed state the slow manifolds are reduced and the limit cycle becomes asymmetric. $S M$ is the stable part of the slow manifold.

1. Choose $c=-2.2, d=0.03, \kappa=0.075$.

Starting the $y$-oscillation near the normal mode plane $y=\dot{y}=0$, the 3-dimensional projection of the solution is rather messy but a projection on the $x-\dot{x}$ plane produces an orbit which seems to fill up a large part of the space taken by the unperturbed orbit; see Fig. 4. Calculation of the Lyapunov exponents of the solution gives the result $\lambda_{1}=0.05205 \ldots, \lambda_{2} \approx 0, \lambda_{3}=-0.13449 \ldots, \lambda_{4}=-13.14938 \ldots$.

There are various ways to characterize the fractal character of a limit set, see for instance [11]. As rather typical we have chosen the Kaplan-Yorke dimension which follows immediately from the Lyapunov exponents. This leads us to a conclusion that we have a chaotic attractor, with the corresponding Kaplan-Yorke dimension near 2.3. Fig. 5 shows the projection of the 3-dimensional Poincaré section of the orbit in $\dot{x}-y$ plane, taking $x=0.5$ as the section (the vertical line in Fig. 4). The projection fits with the previous calculation that the attractor has a dimension larger than 2.

2. Consider the same dynamics, $c=-2.2, d=0.03, \kappa=0.075$, but starting at $y(0)=3, \dot{y}(0)=0.1$ we have oscillations so that $y(t)$ takes alternating values above and below $0.67 \ldots$ Leaving out the transient we find a periodic limit set illustrated in Fig. 6; this is a projection in 3-dimensional space. Projecting the limit set on the $x, \dot{x}$-plane we find a strongly perturbed relaxation oscillation, see Fig. 7. For comparison the unperturbed relaxation oscillation (coupling $c=0)$ is indicated by dots.

Thus, we have at least two attractors.

3. For certain parameter values we find unbounded solutions. We discard these cases as they may correspond with a break-down of the model. 


\section{A simplified model}

Another possibility to clarify the dynamics is to replace system (14) by the equation

$$
\ddot{x}+x=\mu\left(1-x^{2}\right) \dot{x}+\mu c \dot{x} \cos ^{2} q t .
$$

This equation might be illustrative for the behaviour of the relaxation oscillation in the special case when the solutions for $y$ are $2 \pi / q$-periodic. At the same time it is a model of the van der Pol relaxation oscillator with parametric excitation added.

Interesting phenomena arise when varying $c$, see Figs. 8-10. Choosing $c=-16$ we have periodic limiting behaviour; near $c=-18$ it is not clear whether the attractor is (long-) periodic or not periodic. This behaviour corresponds with a small window in parameter space as for $c=-19$ we have again periodic behaviour with two periodic attractors; see Fig. 10. In all cases we observe quenching of the van der Pol relaxation oscillation.

If we increase $c$ above -16 or if we decrease $c$ below -19 the quenching is diminished.

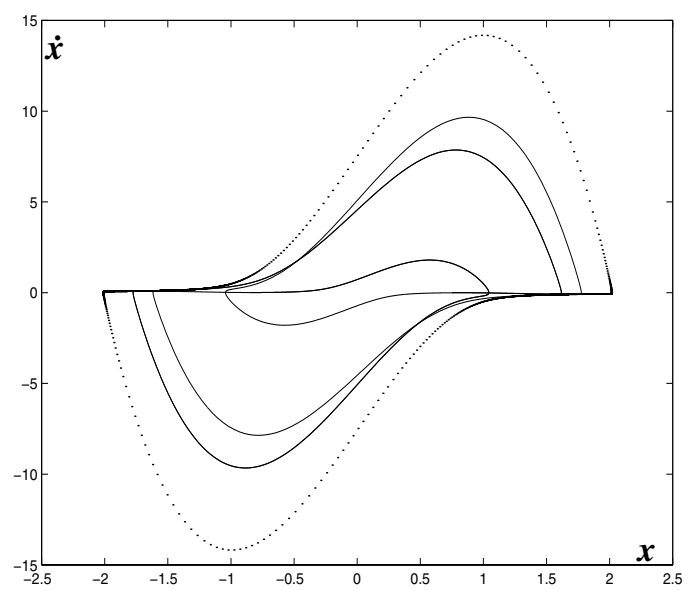

Fig. 8 A limit set of system (16) for $\mu=10, c=-16$. The limit set contains one periodic orbit. The relaxation oscillation corresponding with $c=0$ is dotted.

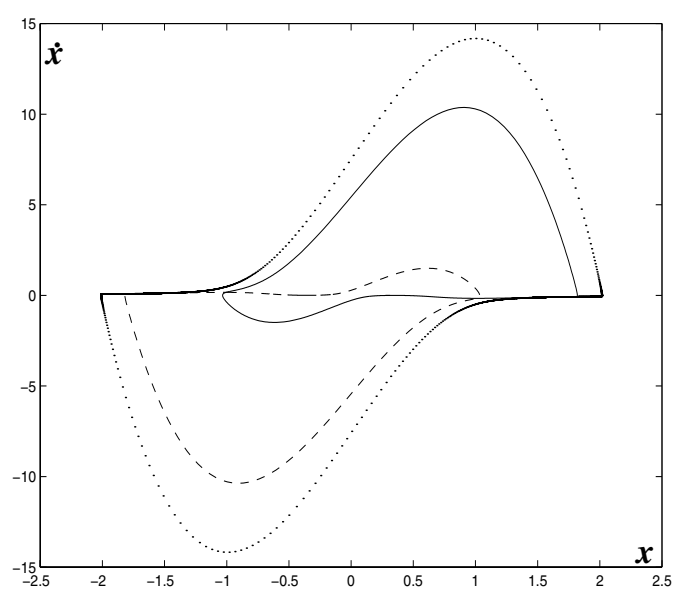

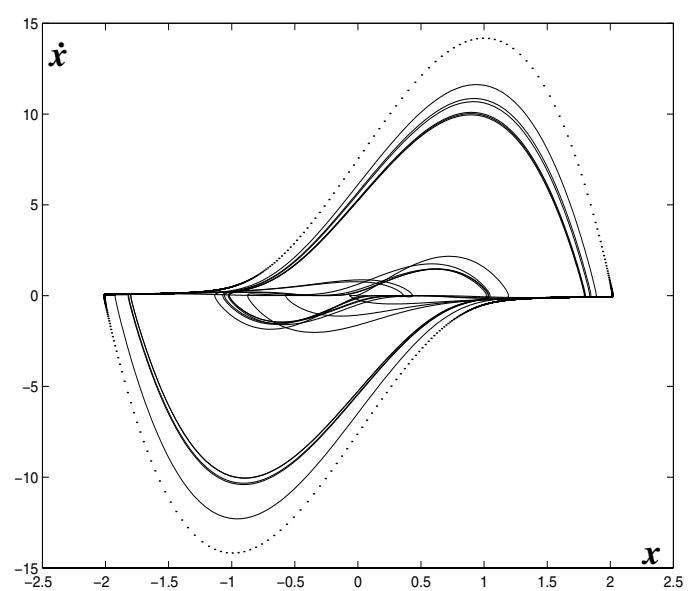

Fig. 9 A limit set of system (16) for $\mu=10, c=-18.1$. The limit set is long-periodic or aperiodic. The relaxation oscillation corresponding with $c=0$ is dotted.
Fig. 10 A limit set of system (16) for $\mu=10, c=-19$. The limit set contains two periodic orbits, both attracting (one is indicated by a full line and the other by dashes). The relaxation oscillation corresponding with $c=0$ is dotted.

\section{Discussion}

The most important conclusion is that to quench relaxation oscillations, apart from the usual tuning conditions, we have to choose the interaction such that strong deformation of the slow manifolds is possible.

In the case that the normal mode relaxation oscillation is destabilised, a number of different limit sets are possible.

The numerical experiments show some interesting results. We obtain a chaotic attractor coexisting with a stable periodic solution. The results also show the effectiveness of the coupling to deform the relaxation oscillation of the system. Related results seem to arise for the simplified model in Sect. 10. 
In the case of coupled oscillators the jumps from one part of the slow manifold to another part can become quite unpredictable and chaotic. More analysis and experiments are needed to understand qualitatively and quantitatively these phenomena.

Acknowledgements The authors would like to thank J. M. Tuwankotta for a correction in the earlier version of this paper. T. Bakri gave technical advice for some numerical experiments in Sect. 9.

\section{References}

[1] Abadi, Nonlinear Dyn. 24, 147-166 (2001).

[2] M. Cartmell, Introduction to Linear, Parametric and Nonlinear Vibrations (Chapman and Hall, London, 1990).

[3] N. Fenichel, Ind. Univ. Math. J. 21, 193-225 (1971).

[4] N. Fenichel, Ind. Univ. Math. J. 23, 1109-1137 (1974).

[5] N. Fenichel, Ind. Univ. Math. J. 26, 81-93 (1977).

[6] N. Fenichel, J. Differ. Equ. 31, 53-98 (1979).

[7] J. Grasman, Asymptotic Methods for Relaxation Oscillations and Applications (Springer-Verlag, New York, 1987).

[8] T. J. Kaper, An introduction to geometric methods and dynamical systems theory for singular perturbation problems, in: Proceedings of the Symposia Appl. Math, AMS 56: Analyzing multiscale phenomena using singular perturbation methods, edited by Jane Cronin and Robert E. O’Malley, Jr. (American Mathematical Society, Providence, Rhode Island, 1999), pp. 85-131.

[9] G. Schmidt and A. Tondl, Non-linear Vibrations (Akademie-Verlag, Berlin, 1986).

[10] A. Tondl, T. Ruijgrok, F. Verhulst, and R. Nabergoj, Autoparametric Resonance in Mechanical Systems (Cambridge University Press, New York, Cambridge, 2000).

[11] F. Verhulst, Nonlinear Differential Equations and Dynamical Systems (Springer-Verlag, New York, 2000).

[12] F. Verhulst, Normal mode instability and quenching, in: Proceedings of the 6th Int. Conference on Vibration Problems, edited by J. Sklíba and B. Marvalová (Liberec University, Czech Republik, 2003), 88-89.

\section{Book Review}

Andrzej Sluzalec, Theory of Metal Forming Plasticity. Classical and Advanced Topics. Springer-Verlag Berlin Heidelberg New York. 2004, XI + 278 pp. Hardcover EUR 106.95*, SFR 169.00, £77.00, US\$ 129.00 ISBN 3-54040648-4 (" gültig in Deutschland incl. MWSt.)

After the publication of "Introduction to Nonlinear Thermomechanics" in 1992 the author presents a second book based on General Continuum Mechanics, but devoted to industrial applications. Since the first book deals with various material behaviour models now the main attention is paid to plasticity.

The textbook is divided into eleven parts. The first part (Deformation of Metallic Body) is an introduction to the basics in Continuum Mechanics since the deformation description and the stress tensor are presented. Here the author has used the traditional concepts and he avoids any details. The second part is devoted to the Thermodynamics of Metal Forming Processes. As a result the governing equations are presented and the temperature field in materials is discussed. The third part deals with various models of the constitutive behaviour. The starting point is the stress-strain diagram, after that the constitutive equations and the evolution equations are presented. Part IV is not included in classical textbooks on plasticity: Powder forming plasticity. Here the author presents some basic ideas for porous materials (the powder material is represented by a porous material with empty spaces and the ratio of the volume of the porous material must be taken into account). In Part $\mathrm{V}$ a short introduction into viscoplasticity is given.
Since in metals surfaces of discontinuity exist the physical laws of conservation on the discontinuities are derived and discussed in Part VI. Such a part is mostly not included into text books on Continuum Mechanics. Part VII (Numerical Solution Methods), Part VIII (Sensitivity in Metal Forming Plasticity) and Part IX (Stochastic Metal Forming Process) are devoted to applications. For the solution of problems one has to use numerical methods. Since the problems are highly non-linear step-by-step methods are discussed. In addition, the sensitivity analysis must be performed and the randomness of the material parameters for example must be taken into account.

The two last parts are devoted to "Contact and Friction" and "Simplified Equations". Contact and friction are important because the workpiece in metalworking processes is deformed by contact. Simplified equations are helpful for first estimates since they allow analytical solutions.

The textbook can be recommended to graduate students and researchers working on problems in metal forming plasticity. It can be used both by beginners and by advancers because all basic knowledge that is necessary for understanding this textbook is presented in the first part of the book. With the help of the references a further reading is possible.

Halle

Holm Altenbach 